\title{
Correction to: The Rim101 pathway mediates adaptation to external alkalization and altered lipid asymmetry: hypothesis describing the detection of distinct stresses by the Rim 21 sensor protein
}

\author{
Keisuke Obara $^{1}{ }^{\circledR} \cdot$ Takumi Kamura $^{1}$
}

Published online: 16 January 2021

๑) Springer-Verlag GmbH Germany, part of Springer Nature 2020

\section{Correction to: Current Genetics \\ https://doi.org/10.1007/s00294-020-01129-0}

In the original publication of the article, the article title was mistakenly published as "Perspective" the correct title should read as "The Rim101 pathway mediates adaptation to external alkalization and altered lipid asymmetry: hypothesis describing the detection of distinct stresses by the Rim 21 sensor protein".

The title has been corrected in this paper.

Publisher's Note Springer Nature remains neutral with regard to jurisdictional claims in published maps and institutional affiliations.

The original article can be found online at https://doi.org/10.1007/ s00294-020-01129-0.

Keisuke Obara

obara.keisuke@g.mbox.nagoya-u.ac.jp

$\triangle$ Takumi Kamura

z47617a@nucc.cc.nagoya-u.ac.jp

1 Division of Biological Science, Graduate School

of Science, Nagoya University, Furo-cho, Chikusa-ku,

Nagoya 464-8602, Japan 